\title{
Trans-scale Coupling in Multiscale Simulations
}

\author{
Feng Rong, Haiying Wang, Mengfen Xia, Fujiu Ke, E Yilong Bai*
}

LNM, Institute of Mechanics, Chinese Academy of Sciences, Beijing 100080, P. R. China

\begin{abstract}
Trans-scale coupling plays a significant role in multiscale problems. Since the mechanisms governing the trans-scale coupling vary from case to case, to identify and characterize the governing mechanisms of trans-scale coupling are the most crucial points in multiscale simulations. The failure of solid media is a typical multiscale process. This paper chooses two model problems, i.e., damage localization in spallation of an Al alloy and the catastrophe transition in a rock under quasi-static loading, to illustrate the trans-scale coupling in different phases of material failure. In the spallation process the governing mechanism of trans-scale effects is the coupling and competition between dynamics at different levels, which can be effectively characterized by two imposed Deborah numbers. In the catastrophe failure of heterogeneous media the governing mechanism of trans-scale coupling is the strong and sensitive coupling between the nonlinear dynamics and the disordered heterogeneity. In addition, the inverse cascade of damage evolution magnifies the effects of microstructures on failure and induces trans-scale sensitivity. Although the concept of critical sensitivity seems to be promising in catastrophe prediction, novel concepts and numerical schemes are still badly needed.
\end{abstract}

\section{KEY WORDS}

multiscale simulation, spallation, catastrophe, trans-scale coupling

*Address all correspondence to baiyl@lnm.imech.ac.cn, Phone (86-10) 62548133 


\section{INTRODUCTION}

In the past several decades, there has been an explosive growth of interest in problems involving multiple space and time scales [1-4] Generally speaking, multiscale problems are very ubiquitous in nature. These problems can be categorized into three classes related to equilibrium, near-equilibrium, and far from equilibrium cases, respectively. Among them, problems in far from equilibrium cases are the greatest challenges.

For multiscale problems in far from equilibrium cases the physical mechanisms or the dynamics may differ on different scales [5], and the interaction between different scales is usually strong and/or sensitive [6]. As a result, the similar solutions in mechanics, which root in identical physics at various scales, are questionable in these problems. In addition, it is inappropriate to deal with an isolated scale of interest, ignoring all other scales. The perturbation method suitable for the weak coupling condition also fails in dealing with strong coupling effects. Therefore what is a suitable method for these problems?

The advances in computer technology have enabled us to exploit multiscale problems with numerical simulation [7]. Many numerical tools focus primarily on the simulation of the global or macroscopic response and are therefore unable to reproduce critical small-scale features and processes that influence the overall structural behavior. On the other hand, direct numerical simulation on a small scale, e.g., molecular dynamics, are most often beyond current capacities and are prohibitive in real applications. Hence many novel numerical schemes, which combine atomistic and continuum simulation, have been proposed to deal with the multiple scales in the problems [810]. However, the computation is governed basically by the smallest time scale in the system, i.e., atomic vibration on the order of fem- toseconds $\left(10^{-15} \mathrm{~s}\right)$. Even if the spatial coupling is truly seamless, difficulties caused by such a small time scale remain unresolved. For more realistic simulations, multiscale algorithms have yet to be developed for both length and time scales in balance.

The failure of solid materials is such a typical process, involving trans-scale coupling of many space and time scales. Our previous studies show that the failure of solids has three phases on different scales: (i) globally stable accumulation of microdamage; (ii) damage localization; and (iii) catastrophic rupture $[11,12]$ In this paper we use two model problems, i.e., damage localization in an $\mathrm{Al}$ alloy under impact loading and the catastrophe transition in a rock under quasi-static loading, to illustrate the transscale coupling in the second and third phases, respectively. Although the mechanism and characterization of trans-scale coupling varies in different cases, there exist some common points, which are noticeable and valuable for the development of new multiscale algorithms.

\section{DAMAGE LOCALIZATION IN AN AL ALLOY UNDER IMPACT LOADING}

We consider a problem of damage evolution owing to the impact of a flying plate of thickness $L$ with velocity $v_{f}$ striking on a target plate, i.e., spallation. Spallation is a typical process with coupled multiple space and time scales [13]. For the time-dependent damage process, which consists of microcrack nucleation and growth, Table 1 lists all parameters involved in the problem, with their corresponding dimensions $L, T$, and $M$ are the notations of the dimensions of length, time, and mass, respectively. Note that for simplicity the constant ratio of flying and target plate is assumed in this paper.

In light of $\pi$ theorem in dimensional analysis, these meso- and macroscopic parameters 
TABLE 1. Parameters and their dimensions

\begin{tabular}{|l|l|l|}
\hline Entity & Notation & Dimension \\
\hline \multicolumn{2}{|c|}{ Macroparameters } \\
\hline sample size & $L$ & $\mathrm{~L}$ \\
\hline material density & $\rho$ & $\mathrm{M} \mathrm{L}^{-3}$ \\
\hline sound speed & $a$ & $\mathrm{~L} \mathrm{~T}^{-1}$ \\
\hline constitutive stress (like yield strength) & $\sigma_{Y}$ & $\mathrm{M} \mathrm{L}^{-1} T^{-2}$ \\
\hline impact velocity & $v_{f}$ & $\mathrm{~L} \mathrm{~T}^{-1}$ \\
\hline \multicolumn{2}{|c|}{ Mesoparameters } \\
\hline nucleation rate of microcrack density & $n_{N}^{*}$ & $\mathrm{~L}^{-4} \mathrm{~T}^{-1}$ \\
\hline growth rate of microcrack & $V^{*}$ & $\mathrm{~L} \mathrm{~T}^{-1}$ \\
\hline microcrack size & $c^{*}$ & $\mathrm{~L}$ \\
\hline
\end{tabular}

in Table 1 form five independent dimensionless numbers. They are as follows:

Mach number

$$
M=\frac{v_{f}}{a}
$$

Damage number

$$
S=\frac{\sigma_{Y}}{\rho a v_{f}}
$$

Imposed Deborah number

$$
D e^{*}=\frac{a c^{*}}{L V^{*}}
$$

Intrinsic Deborah number

$$
D^{*}=\frac{n_{N}^{*} c^{* 5}}{V^{*}}
$$

Length ratio

$$
R=\frac{c^{*}}{L}
$$

Of course, the ratio of $D e^{*}$ to $D^{*}$ forms the other imposed Deborah number $D e=$ $D e^{*} / D^{*}=a / L n_{N}^{*} c^{* 4}$. Of the three Deborah numbers, only two are independent. In addition, the damage numbers [14] include no parameters related to damage and indicate the significance of wave loading $\left(\rho a v_{f}\right)$ in comparison with solid yielding strength $\left(\sigma_{Y}\right)$.

Roughly speaking, the six numbers can be cataloged into three groups. The Mach number $M$ and damage number $S$ are the representation of macroscopic material properties and imposed loading. The intrinsic Deborah number $D^{*}$ represents the mesoscopic kinetic of microdamage evolution. The length ratio $R$ and the two imposed Deborah numbers $D e$ and $D e^{*}$ are all trans-scale dimensionless parameters In particular, the trans-scale Deborah numbers De and $D e^{*}$ are closely related to mesoscopic kinetics of microdamage evolution and macroscopic external loading.

In order to understand the role of these dimensional numbers in spallation more accurately, we should turn to governing equations of the damage evolution [15]. For the spallation process, four equations, the continuum equation, the momentum equation, the constitutive equation, and the damage evolution equation, should be associated as

$$
\begin{gathered}
\frac{\partial \varepsilon}{\partial \bar{T}}=M \frac{\partial \bar{v}}{\partial \bar{X}} \\
\frac{\partial \bar{v}}{\partial \bar{T}}=S \frac{\partial \bar{\sigma}}{\partial \bar{X}}
\end{gathered}
$$




$$
\begin{array}{r}
S M d \bar{\sigma}=[(1-D) d \varepsilon-\varepsilon d D] \\
\frac{\partial D}{\partial \bar{T}}+M \frac{D}{1+\varepsilon} \frac{\partial \bar{v}}{\partial \bar{X}}=\bar{f} \\
=\frac{1}{D e}\left[\bar{f}_{N}(\bar{\sigma})+\bar{f}_{V}\left(\bar{\sigma}, \frac{\bar{T}}{D e^{*}}\right)\right]
\end{array}
$$

where $\varepsilon$ is the strain, $\bar{T}=a T / L$ and $\bar{X}=X / L$ are dimensionless temporal and spatial independent variables, respectively, $\bar{v}=v / \nu_{f}$ and $\bar{\sigma}=\sigma / \sigma^{*}$ are dimensionless velocity and stress, respectively, $D$ is continuum damage, and $\bar{f}$ is the dimensionless dynamic function of damage (DFD), which is the agent bridging macroscopic mechanics and mesoscopic kinetics of damage. Depending on mesoscopic kinetics of microdamage and numerical facility, $\bar{f}$ can be expressed in various forms, such as shown in Eq. (9). For more details of DFD, one can refer to Bai et al. [13] and Davison and Stevens [15]. $M, S, D e$, and $D e^{*}$ are four dimensionless parameters, as introduced above.

Contrary to common sense, the ratio of length scales on meso- and macrolevels $R=$ $c^{*} / L$ does not appear in the governing equations. This looks very abnormal at first sight. Actually, the length ratio $R$ has been combined with time scales to form the dimensionless numbers $D e$ and $D e^{*}$, owing to the nature of the damage evolution problem. The imposed Deborah number $D e^{*}$ is a combination of two ratios: the size-scale ratio $c^{*} / L$ and the ratio of two velocities $V^{*} / a$. In addition, $D e^{*}=t_{V} / t_{i m}$ refers to the ratio of microdamage growth time scale $t_{V}=c^{*} / V^{*}$ over the macroscopically imposed time scale $t_{i m}=L / a$. Hence it represents the competition and coupling between the macroscopically imposed wave loading and the intrinsic microdamage growth Similarly, $D e=t_{N} / t_{i m}$ refers to the ratio of microdamage nucleation time scale $t_{N}=\left(n_{N}^{*} c^{* 4}\right)^{-1}$ over the macroscopically imposed time scale $t_{i m}=L / a$. Hence De represents the competition and coupling between the macroscopically imposed wave loading and the intrinsic microdamage nucleation.

We solved Eqs. (6)-(9) numerically with the finite difference method and studied the effects of dimensionless parameters on damage evolution in the target plate. Now that the effects of the macroscopic numbers $M$ and $S$ on damage evolution are very trivial and have been well documented in literature, emphasis is put on the effects of two trans-scale parameters, De and $D e^{*}$.

At a fixed time, along the axis $\bar{X}$ in the target, there is a damage profile $D(\bar{X})$. In order to depict damage evolution simply and clearly, the effect of imposed Deborah number De on the evolution of maximum damage in the target plate, $D_{\max }=\max \{D(\bar{X})$, for a given $\bar{T}\}$, is shown in Fig. 1. In these calculations, Mach number $M$, damage number $S$, and the imposed Deborah number $D e^{*}$ are identical for all curves. Obviously, the maximum damage in the target plate increases with decreasing $D e$. According to the definition, $D e=t_{N} / t_{i m}$, the decrease of De speeding up the process of damage evolution implies that a shorter microdamage nucleation time scale leads to higher dam-

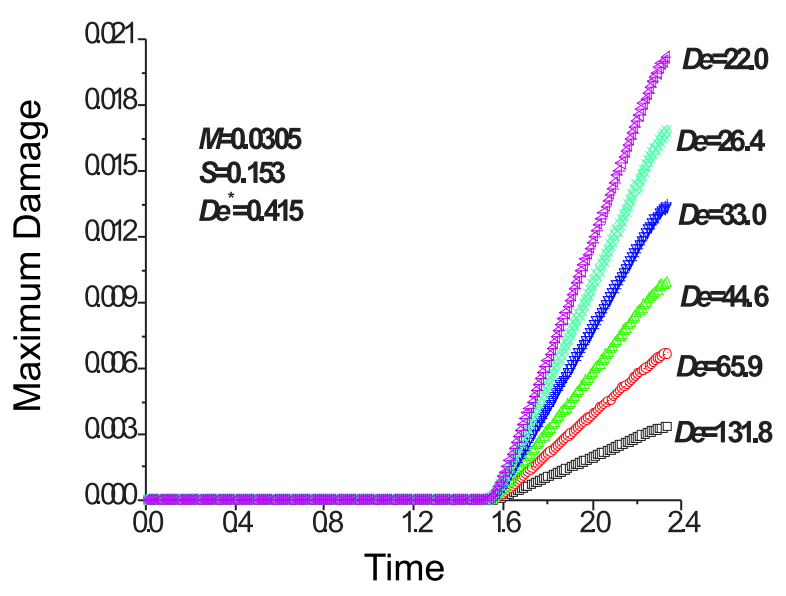

FIGURE 1. Effects of De on the evolution of maximum damage in target plate $(M=0.0305, S=0.153$, $\left.D e^{*}=0.415\right)$ 
age. The nearly linear relation of $D$ versus $\bar{T}$ is mainly owing to the time-independent nucleation rate of microdamage, $\bar{f}_{N}(\bar{\sigma})$.

Figure 2 illustrates the effects of imposed Deborah number $D e^{*}$ on the evolution of maximum damage in the target plate. Similarly to the effect of $D e$, the maximum damage in the target plate increases with $D e^{*}$ decreasing. According to the definition, $D e^{*}=t_{V} / t_{i m}$, the decrease of $D e^{*}$ accelerating the damage evolution process implies that a shorter microdamage growth time scale leads to higher damage. The "explosive" increase of $D$ for smaller $D e^{*}$ results from the time-dependent growth rate of microdamage, $\bar{f}_{V}\left(\bar{\sigma}, \bar{T} / D e^{*}\right)$. This is a reflection of the compound damage noted by Davison and Stevens [16].

Then, what is the crucial difference between the effects of the two imposed Deborah numbers $D e$ and $D e^{*}$ ? Our further simulations show that $D e^{*}$ affects damage localization behavior significantly Fig. 3 shows the normalized accumulated damage distribution at a fixed time. The damage is normalized by its corresponding maximum damage in the target plate. It is evident that damage distributes heterogeneously in the plate. In particular, when the imposed Deborah number $D e^{*}$ decreases, the damage

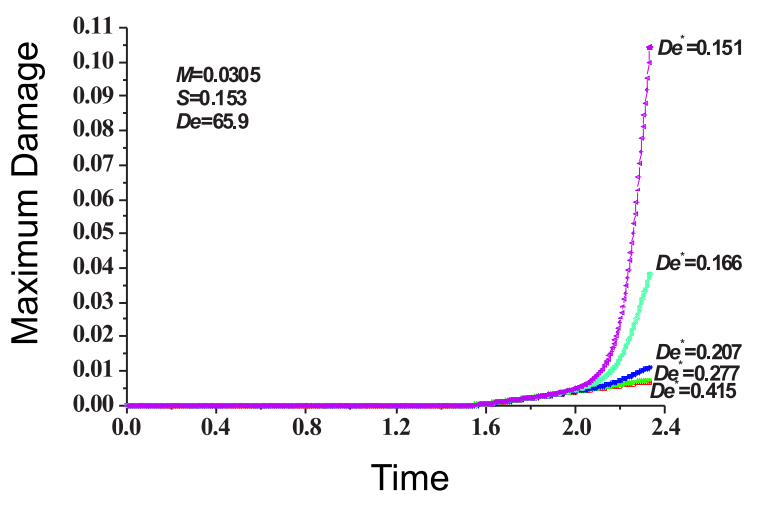

FIGURE 2. Effect of $D e^{*}$ on the maximum damage evolution in the target plate $(M=0.0305, S=0.167$, $D e=65.9$ )

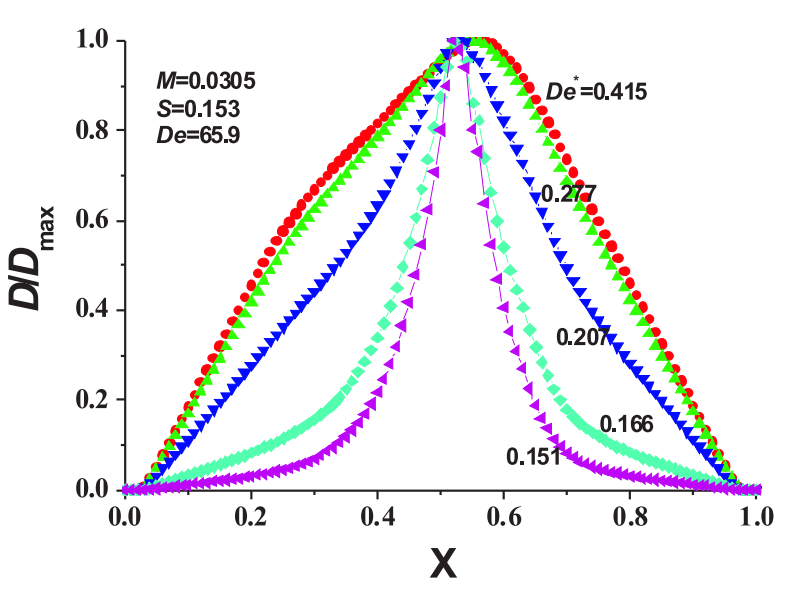

FIGURE 3. Effects of $D e^{*}$ on damage localization $(M=0.0305, S=0.153, D e=65.9, \bar{T}=2.33)$

gets more localized in the plate. However, in contrast to the marked difference in Fig. 3, the simulations show that the mere variation of $D e$, namely the mere variation of the microdamage nucleation rate, does not affect damage localization behavior at all, as shown in Fig. 4.

Obviously, the damage evolution and localization in spallation result from the trans-scale coupling. Physically speaking, there are three kinetic processes at two levels involved in spallation: the macroscopic impact loading, the nu-

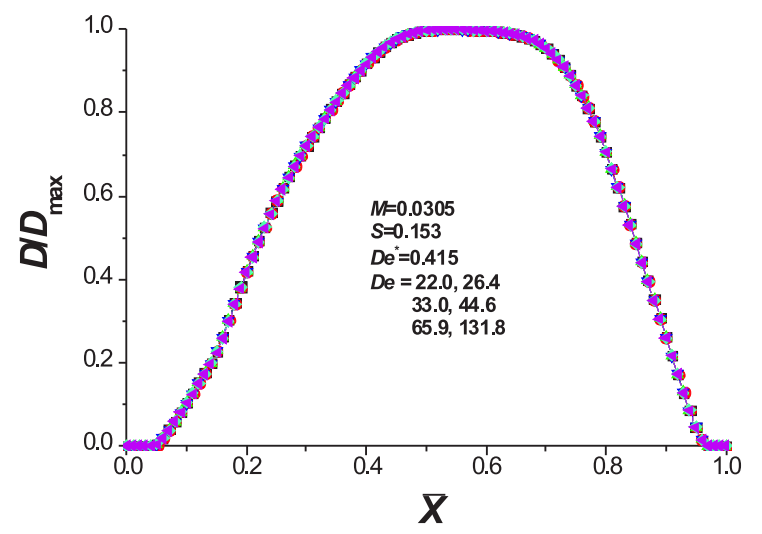

FIGURE 4. Effects of De on damage localization $(M=0.0305, S=0.153, D e=65.9, \bar{T}=2.33)$ 
cleation of microdamage, and the growth of microdamage. The imposed Deborah numbers $D e=a / n_{N}^{*} c^{* 4 L}=t_{N} / t_{i m}$ and $D e^{*}=$ $a c^{*} / L V^{*}=t_{V} / t_{i m}$ essentially root in the transscale nature of spallation. Moreover, they are related to the competition and coupling between damage evolution processes at different scales. As mentioned before, $D e$ and $D e^{*}$ are related to the coupling between the process of macroscopic loading and the process of microdamage nucleation and the coupling between the process of macroscopic loading and microdamage growth, respectively. For a given impact loading time, smaller De means a higher microdamage nucleation rate, and smaller $D e^{*}$ physically means faster microdamage growth. Therefore either smaller $D e$ or $D e^{*}$ results in more damage in the plate. This is qualitatively consistent with numerical results (Figs. 1 and 2). Alternatively, the thicker the sample size $L$, the smaller $D e$ and $D e^{*}$, and then the more damage. This demonstrates the well-known size effect in spallation.

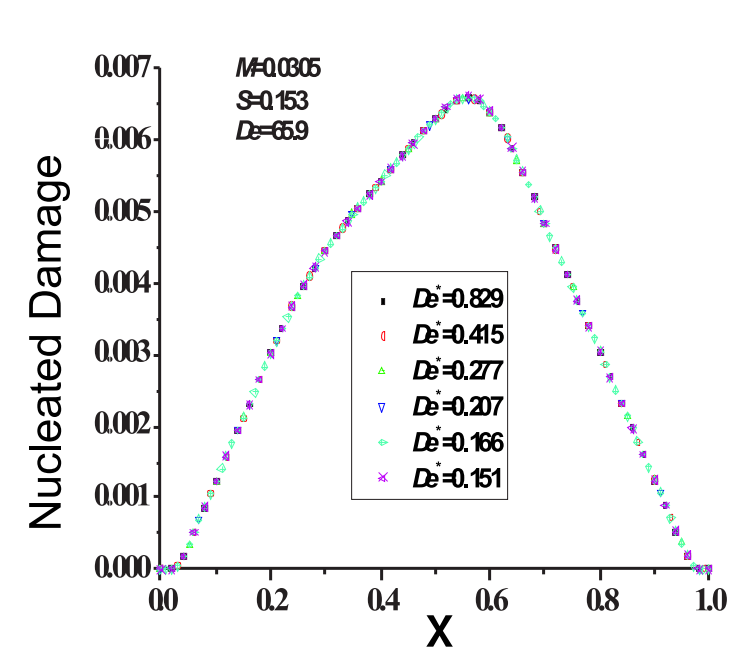

(a) Nucleated Damage v.s. De*
However, the damage localization behavior should be attributed to the imposed Deborah number $D e^{*}$ only. For a given real stress the DFD in the right-hand side of the damage evolution equation (Eq. (9)) are controlled by De and $D e^{*}$, respectively. On the basis of the above discussion on $D e$ and $D e^{*}$, these two terms, $\bar{f}_{N}$ and $\bar{f}_{V}$, correspond to the contribution of microdamage nucleation and growth to macroscopic damage evolution, respectively. We name the accumulated damage contributed by nucleation and growth of microdamage as nucleated damage and grown damage. This is in accord with the concept of simple and compound damage proposed by Davison and Stevens [16]. Therefore the mesoscopic basis of simple and compound damage is nucleated damage and grown damage, respectively.

Figures 5(a) and 5(b) illustrate nucleated damage and grown damage in the target plate, respectively. Figure 5(a) shows that microdamage nucleation results in relatively inhomogeneous distribution of damage, which is caused

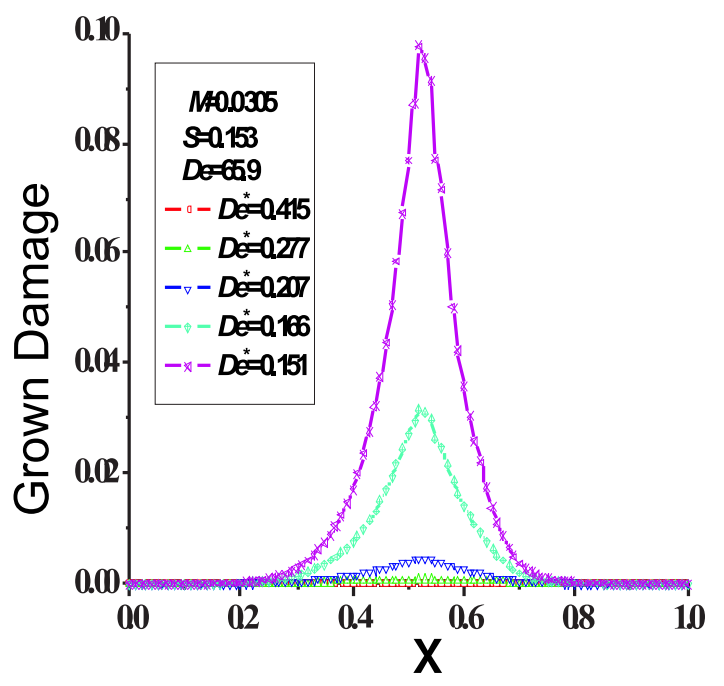

(b) Grown Damage v.s. De*

$$
M=0.0305, S=0.153, D e=65.9, \bar{T}=2.33
$$

FIGURE 5. Variation of nucleated damage, grown damage, and flowed damage with $D e^{*}$ 
by the heterogeneous distribution of stress in the target plate. Since $D<<1$, the history of stress distribution is almost the same for all cases with the same Mach number $M$ and damage number $S$. Therefore the nucleated damage is the same for cases with the same De but different $D e^{*}$ (Fig. 5(a)). However, the grown damage varies significantly for cases with different $D e^{*}$, as shown in Fig. 5(b). Generally, when $D e^{*}$ decreases, the grown damage increases and gets more localized in the target plate. Comparing Fig. 5(a) and Fig. 5(b), we can see that for cases with smaller $D e^{*}$, grown damage is much more localized than nucleated damage. Actually, the term of grown damage serves as a positive feedback in Eq. (9). The heterogeneity of nucleated damage will be amplified by the microdamage growing. The amplification gets stronger with $D e^{*}$ decreasing. Therefore it can be seen that the damage localization is caused by the growth of microdamage. Since the growth of microdamage is controlled by $D e^{*}, D e^{*}$ is closely related to damage localization, and damage is prone to localize in materials with smaller $D e^{*}$.

In one word, in the concerned problems with both macro- and meso- length scales, $L$ and $c^{*}$, the governing mechanism is the trans-scale coupling of three corresponding time scales, which are characterized by two Deborah numbers. To deal with this kind of multiscale problem, much more attention should be paid to time scales, apart from various length scales.

\section{CATASTROPHE TRANSITION IN A ROCK UNDER QUASI-STATIC LOADING}

Damage evolution in heterogeneous media like rocks is a key problem in science. The catastrophe transition from damage accumulation to rupture is the most important feature in the problem. Recent works suggest that the catastrophe transition in rocks can be regarded as a trans-scale process from mesoscopic scale to macroscopic scale, and eventually to global scale, spanning a wide range of space and time scales. Although the formulation in Section 2 can help us deal with damage evolution to localization, it does not provide insight into the very catastrophic rupture of solid media. Hence we turn to numerical simulation to investigate the catastrophic rupture process.

In order to effectively consider the multiscale coupling effects, we established a multiscale finite element model (MFEM). It is actually a multilevel, self-adaptive finite element model In this paper we just give a brief description of the model. More details can be found in Rong et al. [17].

In MFEM, the 4-8 nodes serendipity elements and the linear elastic brittle constitutive relation are adopted. In addition, it is assumed that the damage criterion of a mesoscopic element follows the Coulomb criterion:

$$
F=|\tau|+\mu \sigma-\tau_{S}>0
$$

where $\mu$ is the frictional coefficient and $\tau_{S}$ is the inherent shear strength. In the simulation, if the stress state of an element satisfies this inequality, it will be adaptively refined to four smaller elements, and when an elementary element, i.e., the finest element, does satisfy this inequality, it is assumed to be damaged. In accordance with Eq. (10), the damage of elements may appear at three different directions (Fig. 6(b)) so that the elements can be distinguished as intact, partially damaged, and entirely damaged. Both the initial elastic modulus and the friction coefficient $\mu$ are assumed to be identical for all elementary elements. However, the material heterogeneity is described by varying $\tau_{S}$ of elementary elements, according to a Weibull distribution $h\left(\tau_{S}\right)=m\left(\tau_{S}^{m-1} / \eta^{m}\right) \exp \left[-\left(\tau_{S} / \eta\right)^{m}\right]$.

With this model we studied a rock sample under in-plane pressure (Fig. 6(a)). Both the height and width of the sample are $0.1 \mathrm{~m}$. Its upper surface is the loading surface, while the lower surface is fixed. Although this is a plane 


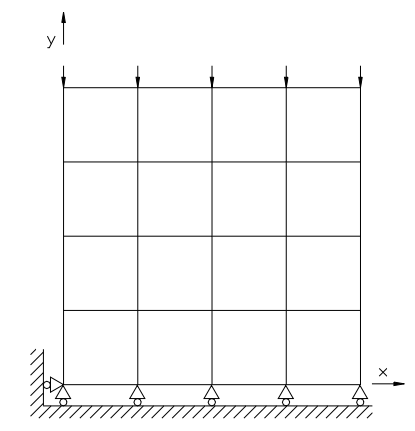

(a)
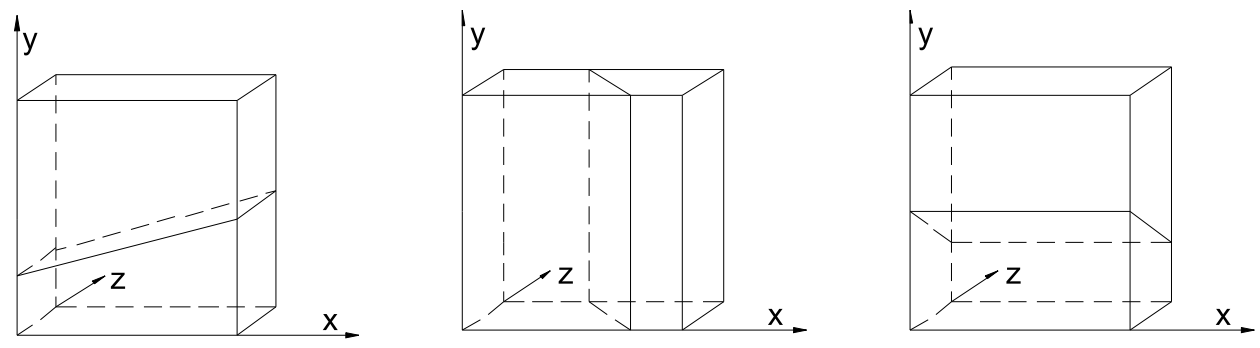

(b)

FIGURE 6. (a) Schematic of the model. (b) Three-dimensional damage in elements

stress problem, three-dimensional damage can also occur on three different orientations in one element, as shown is Fig. 6(b). After an element fails in one direction the elastic modulus in this direction will be reduced.

Similar to the process of spallation, there are several time scales in the failure process of rocks, e.g., external loading time $t_{e x}$, the stress relaxation time $t_{P}$, and the damage relaxation time $t_{D}$. In the simulation the quasi-static loading mode is used, which implies

$$
t_{P}<<t_{D}<<t_{e x}
$$

Inequality (11) physically means that in one loading step the external load remains unchanged until the last damage happens, and stress redistributes if only damage occurs. Ac- tually, the latter condition can be automatically achieved by solving the equilibrium equation of stress. In our simulation the Young's modulus is $E_{0}=47.23 \times 10^{9} \mathrm{~Pa}$, the Poisson's ratio is $v=0.3$, the friction coefficient is $\mu=0.639$, and the parameters of Weibull distribution for inherent shear strength $\tau_{S}$ are $m=3$ and $\eta=$ $3.10 \times 10^{7} \mathrm{~Pa}$, as given by Jarger and Cook [18] for marble. In order to check the mesh effect we compared the results obtained by the selfrefined FE and the FE with the finest meshes only. The error is characterized by the difference between the damages of the two simulations after catastrophe. The damage of the selfrefined FE simulation is $7.15 \%$, and that of the FE simulation with the finest meshes only is $7.37 \%$. The consistency of the two results suggests an acceptable mesh effect. 
Figure 7 shows the nominal stress-strain curve and damage pattern evolution in a sample [19]. At the initial stage (e.g., step 202), damage is relatively uniform. As damage accumulates, damage clusters are formed (e.g., step 502, step 531). At last, the catastrophic rupture happens (step 531).

Let us make a detailed observation on the damage pattern evolution in step 531. In fact, step 531 can be divided into 33 substeps [19]. In each substep the stress is in equilibrium, while damage can still evolve and cause stress to redistribute in the next substep. Figure 8 shows the damage pattern at various substeps in step 531. Obviously, in this step, damage coalescence expands to become the largest damage cluster and causes an inverse cascade of damage from a small scale to a large one, and eventually to a global scale. Hence the catastrophic rupture can mainly be attributed to damage coalescence.

Then, what is the governing mechanism causing the trans-scale damage coalescence and the eventual catastrophic rupture? We supposed that damage evolution leads to stress redistribution, and then stress redistribution can speed up damage coalescence. We define the stress transfer distance as the distance between a newly damaged element in one substep and the element where its Coulomb stress increases in the same substep. Let us compare the stress transfer distance and the stress increment at different phases in the loading process (Fig. 9), in which the stress transfer distance is normalized by $d_{0}$, the dimension of the finestscale element. It is evident that comparing with the damage accumulation phase, much stronger damage-induced stress redistribution occurs during catastrophe transition, which can be characterized with much larger stress increments induced by a damage event within a finite stress transfer distance [19]. Owing to the heterogeneity of the sample, the stress redistribution is a strong nonlinear dynamical process. During catastrophe transition the nonlinear dynamics of stress redistribution interacts

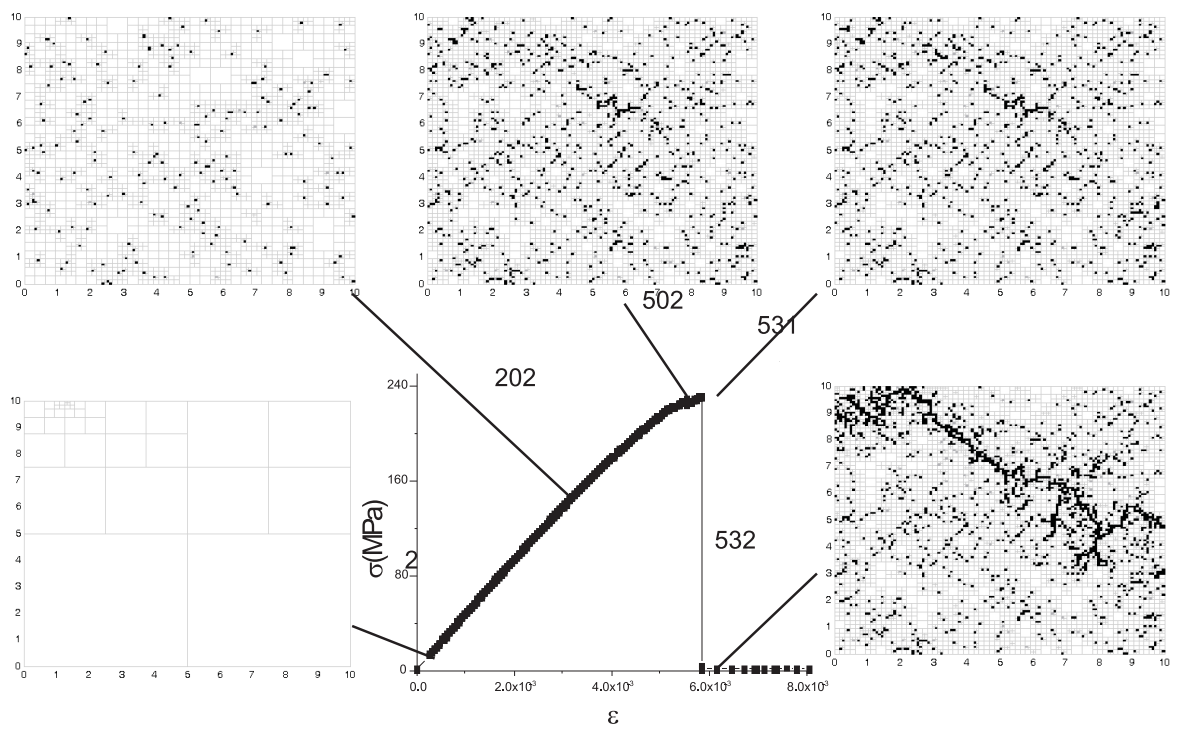

FIGURE 7. The damage evolution patterns. The numbers (like 2, 202, 502, etc.) denote the quasi-static loading steps. In the patterns, there are three kinds of darkness: black indicates entirely damaged; gray indicates partially damaged; and white indicates still intact 


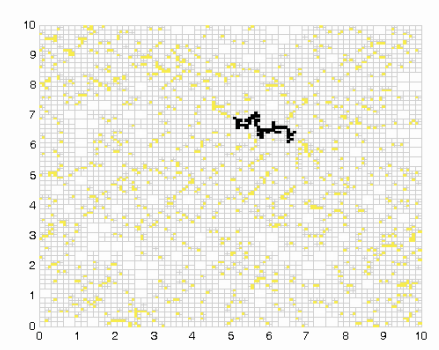

(a) Substep 1

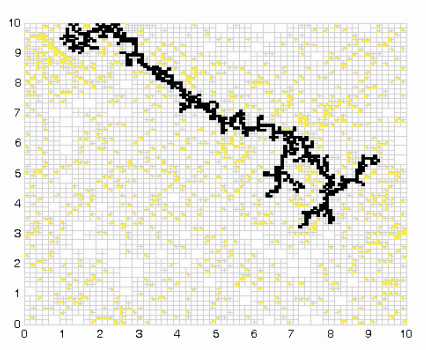

(c) Substep 27

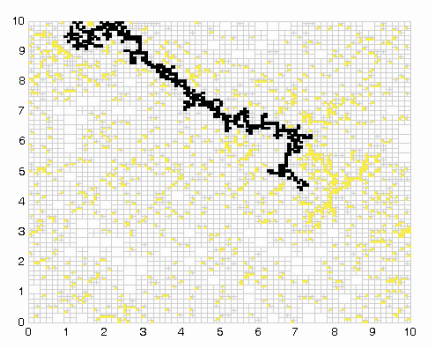

(b) Substep 26

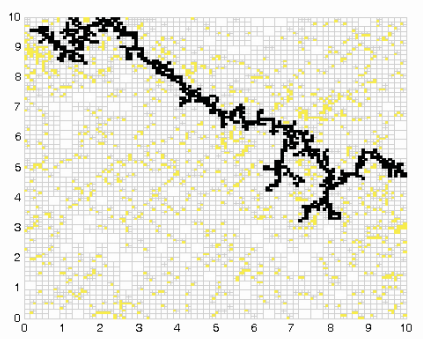

(d) Substep 33

FIGURE 8. The damage coalescence during the catastrophic transition. In the pattern, there are three kinds of elements with different chroma: black indicates the damaged elements forming the largest damage cluster; gray indicates those damaged elements elsewhere; and white elements are still intact

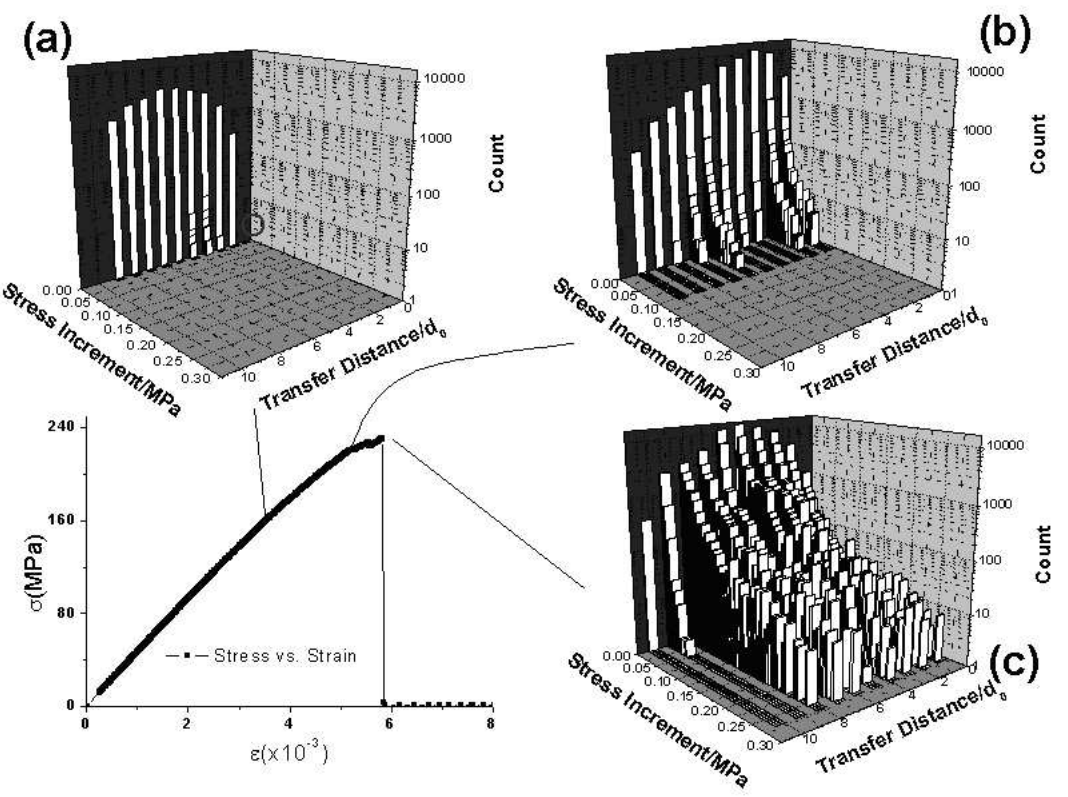

FIGURE 9. Stress transfer distance distribution: (a) the early stage of damage accumulation; (b) the later stage of damage accumulation; (c) the catastrophic rupture 
strongly with the heterogeneity of the sample. The strong interaction causes a very high stress increment in far field of the sample, which leads to damage coalescence, and eventually to catastrophe rupture. Therefore the underlying mechanism of the trans-scale, inverse cascade of damage during the catastrophe transition is the coupling between the dynamical nonlinearity and the disordered heterogeneity.

The coupling between the dynamical nonlinearity and the disordered heterogeneity makes the catastrophe prediction a real challenge for us. Owing to the strong coupling, the catastrophic failure of solids may sensitively depend on some details of mesoscopic heterogeneity. This is a trans-scale sensitivity. In theoretical analysis, trans-scale sensitivity invalidates the global mean field approximation and many homogenization methods. In numerical simulation, trans-scale sensitivity causes mesh sensitivity. Since the trans-scale sensitivity is very tricky to deal with, to develop new concepts of numerical schemes for catastrophe prediction is a real challenge focus.

Finally, we can define a parameter to characterize the trans-scale sensitivity quantitatively. The concept of trans-scale sensitivity means that in heterogeneous brittle media the response of the sample to controlling variables, like external loading, may become significantly sensitive as the sample approaches its catastrophe transition point. There may be various definitions of the response and the sensitivity. Figure 10 shows the variation of the sensitivity defined by energy release versus the boundary displacement in the process. It can be found that there is a significant increase in trans-scale sensitivity $S$ when the sample is approaching its catastrophe transition. Hence if the energy release and the governing variable are both measurable, the sensitivity may provide clues to catastrophe prediction. This concept has been applied to rock tests and earthquake forecasts and looks promising. From the

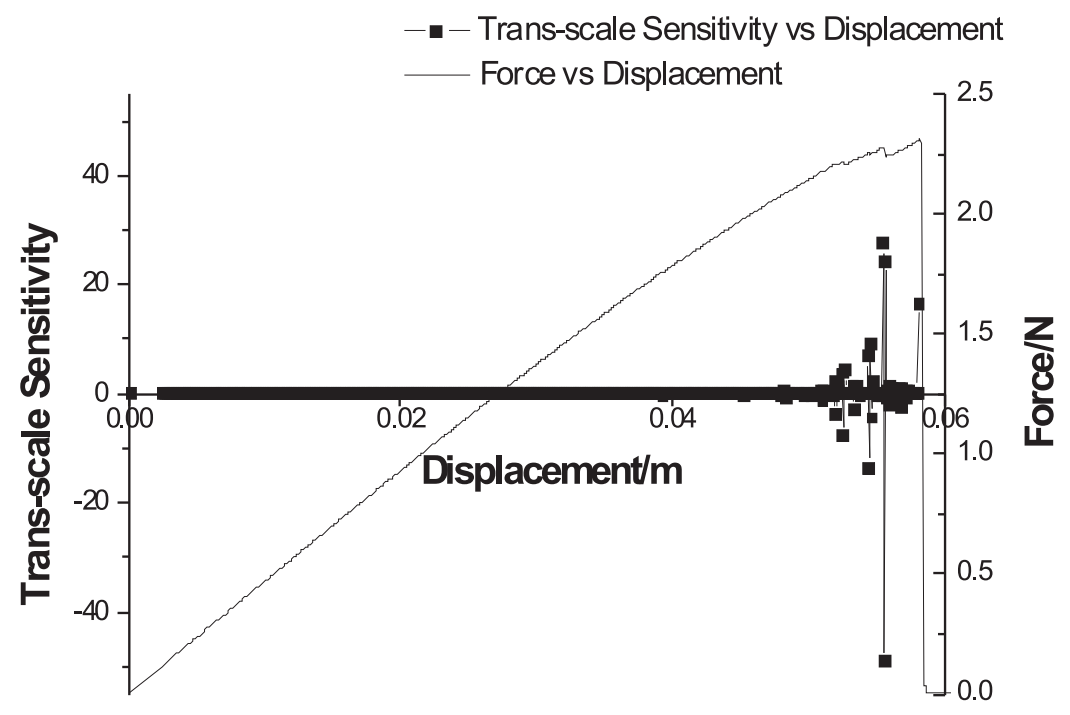

FIGURE 10. Trans-scale sensitivity based on MFEM simulation 
viewpoint of multiscale problems the sensitivity reflects the strong correlation between the extreme events at larger scale and the events at intermediate to small scales. In particular, this implies the significant effect of trans-scale sensitivity in multiscale simulations.

\section{SUMMARY}

Trans-scale coupling plays a significant role in multiscale problems. Since the mechanisms governing the trans-scale coupling vary from case to case, it is truly important to concretely demonstrate the governing mechanisms of trans-scale coupling in various cases. Only after that can we can establish effective theoretical characterizations or numerical schemes for trans-scale coupling effects.

The failure of solid media is a typical multiscale process. This paper chooses two model problems, i.e., damage localization in spallation of an $\mathrm{Al}$ alloy and the catastrophe transition in a rock under quasi-static loading, to illustrate the trans-scale coupling in different phases of material failure.

In the spallation process the governing mechanism of trans-scale effects is the coupling and competition between dynamics at different levels, which can be effectively characterized by the two imposed Deborah numbers.

In the catastrophe failure of heterogeneous media the governing mechanism of trans-scale coupling is the strong and sensitive coupling between the nonlinear dynamics and the disordered heterogeneity. In addition, the inverse cascade of damage evolution magnifies the effects of microstructures on failure and induces trans-scale sensitivity. How to deal with the trans-scale sensitivity might be a big challenge in multiscale simulations. Although the concept of sensitivity seems to be promising in catastrophe prediction, novel concepts and numerical schemes are still badly needed.

\section{ACKNOWLEDGMENT}

This work is granted by the National Natural Science Foundation of China (NSFC 10172084, 10232050, 10372012, and 10302029), the Chinese Academy of Sciences (KJCX2-SW-L2), and Major State Research Project No. G200007735.

\section{REFERENCES}

1. Glimm, J., and Sharp, D. H., Multi-scale science: a challenge for the twenty-first century, SIAM News 30:1-7, 1997.

2. Kwauk, M., and Li, J. H., Three transfers, plus one reaction and multi-scale (in Chinese), Prog. Nat. Sci. 10:1078-1082, 2000.

3. He, G. W., Xia, M. F., Ke, F. J., and Bai, Y. L., Multiscale coupling: challenges and opportunities, Prog. Nat. Sci. 14(6):463-466, 2004.

4. Wang, H. Y., He, G. W., Xia, M. F., Ke, F. J., and Bai, Y. L., Multiscale coupling in complex mechanical systems, Chem. Eng. Sci. 59:1677-1686, 2004.

5. Bai, Y. L., Xia, M. F., Ke, F. J., and Li, H. L., Nonequilibrium evolution of collective microdamage and its coupling with mesoscopic heterogeneities and stress fluctuations, High-Pressure Shock Compression of Solids VI: Old Paradigms and New Challenges, Horie, Y., Thadhani, N., and Davison, L. (eds.), pp. 255-278, SpringerVerlag, Berlin, 2002.

6. Xia, M. F., Ke, F. J., and Bai, Y. L., Threshold diversity and trans-scale sensitivity in a nonlinear evolution model, Phys. Lett. A 236:60-64, 1997.

7. Phillips, R., Crystals, Defects, and Microstructure Modeling Across Scales, Cambridge Univ. Press, Cambridge, 2001.

8. Tadmor, E. B., Ortiz, M., and Phillips, R., Quasicontinuum analysis of defects in solids, Philos. Mag. A 73(6):1529-1563, 1996.

9. Diestler, D. J., Coarse-grained descriptions of multiple scale processes in solid systems, Phys. Rev. B 66(18):184104, 2002.

10. Qian, D., Wagner, G. J., and Liu, W. K., A multiscale projection method for the analysis of car- 
bon nanotubes, Comput. Methods Appl. Mech. Eng. 193(17-20):1603-1632, 2004.

11. Bai, Y. L., Lu, C. S., Ke, F. J., and Xia, M. F., Evolution induced catastrophe, Phys. Lett. A 185:196-200, 1994.

12. Xia, M. F., Ke, F. J., and Bai, Y. L., Evolution induced catastrophe in a nonlinear dynamical model of materials failure, Nonlinear Dyn. 22:205-224, 2000.

13. Bai, Y. L., Bai, J., Li, H. L., Ke, F. J., and Xia, M. F., Damage evolution, localization and failure of solids subjected to impact loading, Int. J. Impact Eng. 24(6-7):685-701, 2000.

14. Johnson, W., Impact Strength of Materials, Edward Arnold, London, 1972.

15. Wang, H. Y., Xia M. F., Ke, F. J., and Bai, Y. L., Microdamage evolution, energy dissipation and its trans-scale effects on macroscopic failure, Mech. Mater., 38(1-2):57-67, 2006.

16. Davison, L., and Stevens, A. L., Continuum measures of spall damage. J. Appl. Phys. 43(10):988-994, 1972.

17. Rong, F., Xia, M. F., Ke, F. J., and Bai, Y. L., Multiscale FEM and Its Application to Damage Evolution of Heterogeneous Brittle Media. In Computational Mechanics WCCM VI in conjunction with APCOM '04, Art. No. 522, Tsinghua Univ. Press \& Springer-Verlag, Beijing, 2004.

18. Jarger, J. C., and Cook, N. G. W., Fundamentals of Rock Mechanics (in press), 3rd ed., Chapman and Hall, London, (in press) 1979.

19. Rong, F., Wang, H. Y., Xia, M. F., Ke, F. J., and Bai, Y. L., Damage coalescence induced catastrophic rupture in heterogeneous brittle media, Pure Appl. Geophys. (in press), 2006. 
